\title{
No association of COVID-19 transmission with temperature or UV radiation in Chinese cities
}

To the Editor:

The coronavirus disease 2019 (COVID-19) epidemic, which was first reported in December 2019 in Wuhan, China, has caused 80904 confirmed cases as of 9 March 2020, with 28673 cases being reported outside of China. It has been declared a pandemic by the World Health Organization (WHO), has exhibited human-to-human transmissibility and has spread rapidly across countries [1]. Although the Chinese government has taken various measures to control city-to-city transmission (e.g. shutting down cities, extending holidays) and many other countries have implemented measures (such as airport screening and testing patients who have reported symptoms), the number of cases is still increasing quickly throughout the world.

Previous studies have shown the importance of weather variables in the transmission of infectious diseases, including, but not limited to, influenza and severe acute respiratory syndrome (SARS). For example, a sharp change of ambient temperature was associated with an increased risk of SARS [2, 3]. Influenza transmission is often enhanced in the presence of cold and/or dry air [4]. In northern Europe, low temperature and low ultraviolet (UV) indexes were correlated with peaks of influenza virus activity during 2010-2018 [5]. Therefore, it is hypothesised that COVID-19 transmission may decrease or even disappear when the temperature and UV radiation increase in the summer. In this study, we aim to determine the association of meteorological factors with the transmission of COVID-19 in various Chinese cities.

We collected COVID-19 confirmed case information in China reported by the National Health Commission (www.nhc.gov.cn/xcs/xxgzbd/gzbd_index.shtml) and the Provincial Health Commissions of China (http://wjw.hubei.gov.cn/bmdt/ztzl/fkxxgzbdgrfyyq/). We used the cumulative number of confirmed cases from 224 cities (207 outside Hubei, 17 inside Hubei) with no less than 10 cases as of 9 March 2020, and calculated the basic reproduction number $\left(\mathrm{R}_{0}\right)$ for 62 cities (50 outside Hubei, 12 inside Hubei) with $>50$ cases as of 10 February 2020 (COVID-19 peak time in China). $\mathrm{R}_{0}$ means the expected number of secondary cases generated by an initial infectious individual, in a completely susceptible population. If $\mathrm{R}_{0}<1$, then the disease-free equilibrium is locally asymptotically stable; whereas, if $\mathrm{R}_{0}>1$, then it is unstable. Thus, $\mathrm{R}_{0}$ is a threshold parameter.

Meteorological data, including daily mean temperature and relative humidity, were collected from the China Meteorological Data Sharing Service System. Regarding UV radiation, daily erythemally weighted daily dose (EDD) data were extracted using the Dutch-Finnish ozone monitoring instrument (OMI) level 2 UV irradiance products, version 003 (OMUVB V003) at $13 \mathrm{~km} \times 24 \mathrm{~km}$ resolution. OMUVB is a satellite-based remote sensing dataset, which was downloaded from earthdata.nasa.gov [6] OMI is a nadir-viewing spectrometer aboard the NASA Aura satellite covering a UV wavelength of 270-380 nm. Average of EDD values from OMI pixels matched within the city area were assigned as the daily mean EDD level for the corresponding city.

We used $\mathrm{R}$ to assess the associations of meteorological factors (including temperature, relative humidity and UV radiation) with the spreadability of COVID-19. In particular, we averaged daily temperature, maximum temperature, minimum temperature, relative humidity and UV radiation (EDD data) from early January to early March for 224 cities. Multiple regression methods were used to explore the association of meteorological factors with cumulative incidence rate and $\mathrm{R}_{0}$ in the same period.

@ERSpublications

There is no association of COVID-19 transmission with temperature or UV radiation in Chinese cities https://bit.ly/2UWOl7I

Cite this article as: Yao Y, Pan J, Liu Z, et al. No association of COVID-19 transmission with temperature or UV radiation in Chinese cities. Eur Respir J 2020; 55: 2000517 [https://doi.org/10.1183/13993003.005172020]. 

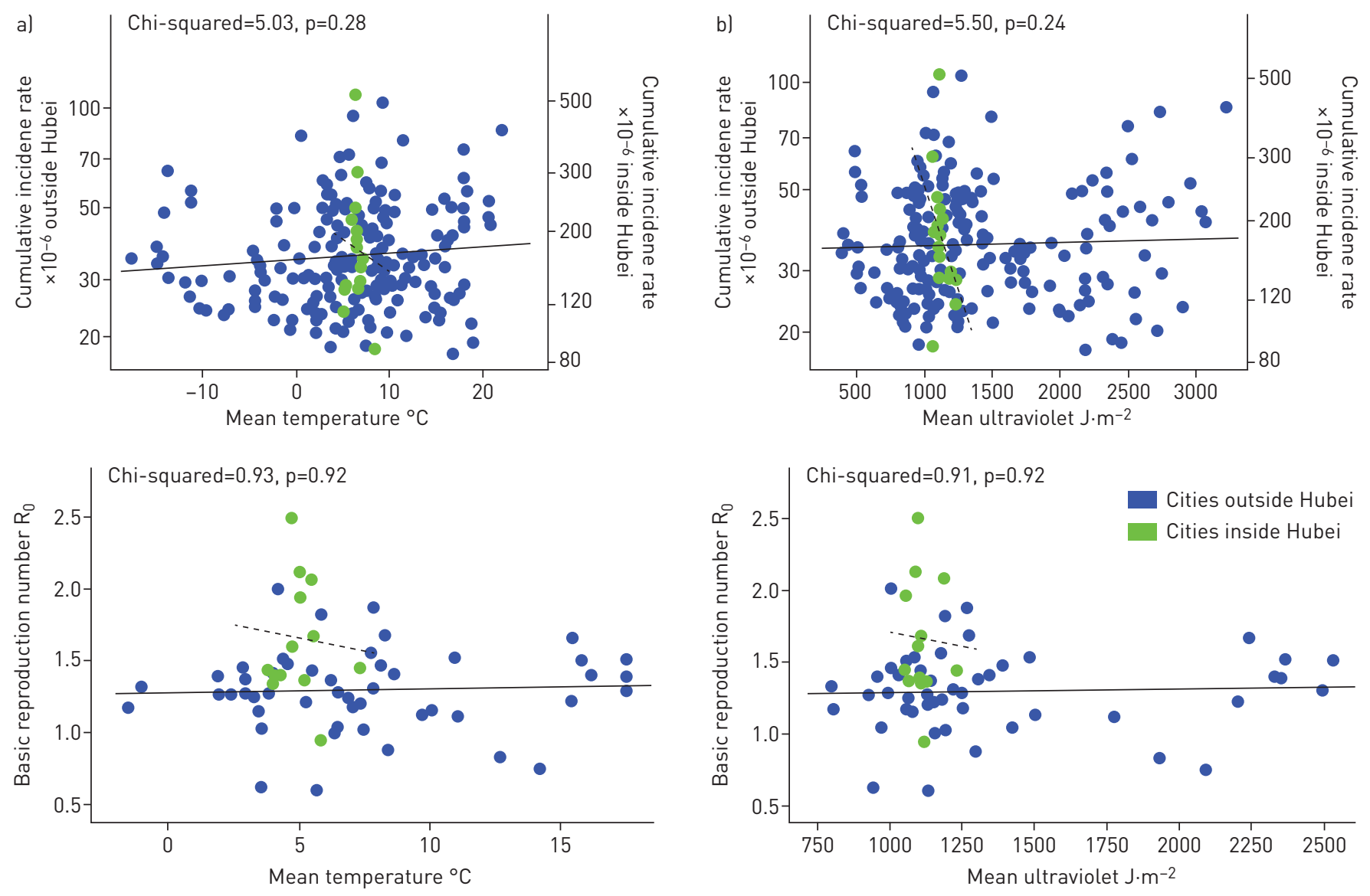

FIGURE 1 Temperature and ultraviolet spreadability of coronavirus disease 2019. a) Cumulative incidence rate and basic reproduction number ( $R_{0}$ ) hold no significant association with the temperature in cities outside and inside Hubei. Relative humidity and ultraviolet effects have been adjusted in the model. b) Cumulative incidence rate and $\mathrm{R}_{0}$ hold no significant associations with ultraviolet in cities outside and inside Hubei. Temperature and relative humidity effects have been adjusted in the model. Solid and dashed lines refer to the linear trend of the correlation of the cumulative incidence rate or basic reproductive number and environmental factors in cities outside Hubei (solid lines) and inside Hubei (dashed lines).

Among the 224 cities, the mean $\pm \mathrm{SD}$ (range) were $5.9 \pm 7.5^{\circ} \mathrm{C}\left(-17.8-22.0^{\circ} \mathrm{C}\right)$ for temperature and 1332.5 $\pm 594.0 \mathrm{~J} \cdot \mathrm{m}^{-2}\left(385.33-3221.96 \mathrm{~J} \cdot \mathrm{m}^{-2}\right)$ for EDD. Temperature and EDD tended to decrease toward high latitude and altitude. The mean \pm SD (range) were $60.3 \pm 324 \cdot 0 \cdot 10^{-6}\left(1.9-4509.1 \cdot 10^{-6}\right.$ ) for cumulative incidence rate in 224 cities and $1.4 \pm 0.3(0.6-2.5)$ for $\mathrm{R}_{0}$ in 62 cities. The top three cities with the highest $\mathrm{R}_{0}$ and the top 15 cities with the highest cumulative incidence rate were all in in the Hubei province.

After adjustment for relative humidity and UV, as shown in figure 1a, temperature held no significant association with cumulative incidence rate (Chi-squared $=5.03, \mathrm{p}=0.28$ ) or $\mathrm{R}_{0}$ (Chi-squared $=0.93, \mathrm{p}=0.92$ ), in cities both outside and inside Hubei, which indicated that the spreadability of COVID-19 would not change with increasing temperature. Similarly, as shown in figure $1 \mathrm{~b}$, UV was not significantly associated with cumulative incidence rate (Chi-squared $=5.50, \mathrm{p}=0.24$ ) and $\mathrm{R}_{0}$ (Chi-squared $=0.91, \mathrm{p}=0.92$ ) after adjustment for temperature and relative humidity, suggesting that the spreadability of COVID-19 would not change with increasing UV exposure. In addition, we did not find significant associations of relative humidity, maximum temperature and minimum temperature with cumulative incidence rate or $\mathrm{R}_{0}$ of COVID-19.

Previous results on the relationship between respiratory-borne infectious diseases and temperature have indicated that both SARS and influenza need to survive under certain temperature conditions, and increasing temperature can reduce their ability to spread $[7,8]$. The underlying hypothesis as to why warmer seasons tend to decrease the spread of viruses include: higher vitamin D levels, resulting in better immune responses [9]; increased UV radiation; and no school in the summer (when children are clustered together, transmission rates of flu and measles increase). Reports of UV and respiratory diseases have also been considered, and previous studies have shown that high levels of UV exposure can reduce the spread of SARS-CoV virus [10].

The results of this study, however, do not follow this expected pattern. According to the current results, the cumulative incidence rate and $\mathrm{R}_{0}$ of COVID-19 held no significant association with ambient temperature, suggesting that ambient temperature has no significant impact on the transmission of 
SARS-CoV-2. This is quite similar to the Middle East respiratory syndrome (MERS) epidemic in the Arabian Peninsula, where MERS cases continued when temperatures were $45^{\circ} \mathrm{C}$ [11]. Other newly emergent zoonotic diseases, such as Ebola or pandemic strains of influenza, have also occurred in unpredictable patterns. Even though the transmission of SARS, which began in November 2002 and ended in July 2003, suggests it might be seasonal, it also might have been controlled by effective case finding, contact tracing and quarantine.

Our study has limitations. First, our study period may not represent a whole meteorological pattern associated with the transmissibility of COVID-19. However, we did not observe reduced transmissibility of COVID-19 in some southern Chinese cities (e.g. Sanya, Haikou and Danzhou) that had average daily temperatures $>20^{\circ} \mathrm{C}$ (maximal temperature $>30^{\circ} \mathrm{C}$ ), suggesting the robustness of our findings. Certainly, further studies with a longer follow-up period and wider temperature range are warranted.

Secondly, given the ecological nature of our study, other city-level factors, such as the feasibility of implementing the COVID-19 control policy, the urbanisation rate and the availability of medical resources, may affect the transmissibility of COVID-19 and confound our findings. Future studies should develop complicated models with a high spatial-temporal resolution to assess the relationship between meteorological conditions and the epidemiological characteristics of COVID-19.

In summary, our study does not support the hypothesis that high temperature and UV radiation can reduce the transmission of COVID-19. It might be premature to count on warmer weather to control COVID-19.

Ye Yao ${ }^{1,2}$, Jinhua Pan $\circledast^{1,2}$, Zhixi Liu ${ }^{1,2}$, Xia Meng ${ }^{1,2}$, Weidong Wang ${ }^{1}$, Haidong Kan $^{1,3}$ and Weibing Wang ${ }^{1,3}$ ${ }^{1}$ School of Public Health, Fudan University, Shanghai, China. ${ }^{2}$ These authors contributed equally to this study. ${ }^{3}$ These authors contributed equally to this study.

Correspondence: Weibing Wang, School of Public Health, Fudan University, Shanghai 200032, China. E-mail: wwb@ fudan.edu.cn

Received: 01 March 2020 | Accepted after revision: 29 March 2020

Author contributions: Ye Yao, Weibing Wang and Haidong Kan designed the study. Jinhua Pan, Zhixi Liu, Ye Yao and Weibing Wang collected COVID-19 incidence data and gained insight into the biology and natural history of the virus. Jinhua Pan, Zhixi Liu, Ye Yao and Weibing Wang developed the model and obtained the related parameters. Weidong Wang and Haidong Kan collected meteorological factors. Ye Yao, Jinhua Pan, Zhixi Liu and Xia Meng drafted the manuscript. Haidong Kan and Weibing Wang revised the manuscript. All authors critically reviewed and approved the final version of the manuscript.

Conflict of interest: None declared.

Support statement: This study was sponsored by the Bill and Melinda Gates Foundation (grant number OPP1216424) and Fudan University research project on the COVID-19 emergency (grant number IDF201007). Funding information for this article has been deposited with the Crossref Funder Registry.

\section{References}

1 Li Q, Guan X, Wu P, et al. Early transmission cynamics in Wuhan, China, of novel coronavirus-infected pneumonia. N Engl J Med 2020; 382: 1199-1207.

2 Tan J, Mu L, Huang J, et al. An initial investigation of the association between the SARS outbreak and weather: with the view of the environmental temperature and its variation. J Epidemiol Community Health 2005; 59: $186-192$.

3 WHO. Coronavirus disease 2019 (COVID-2019) situation report. https://www.who.int/docs/default-source/coronaviruse/ situation-reports/20200227-sitrep-38-covid-19.pdf?sfvrsn=9f98940c_2 Date last accessed: 27 February 2020.

$4 \mathrm{Xu} \mathrm{Z,} \mathrm{Hu} \mathrm{W,} \mathrm{Williams} \mathrm{G,} \mathrm{et} \mathrm{al.} \mathrm{Air} \mathrm{pollution,} \mathrm{temperature} \mathrm{and} \mathrm{pediatric} \mathrm{influenza} \mathrm{in} \mathrm{Brisbane,} \mathrm{Australia.} \mathrm{Environ}$ Int 2013; 59: 384-388.

5 Ianevski A, Zusinaite E, Shtaida N, et al. Low temperature and low UV indexes correlated with peaks of influenza virus activity in Northern Europe during 2010-2018. Viruses 2019; 11: E207.

6 Hovila J, Arola A, Tamminen J. https://disc.gsfc.nasa.gov/datasets/OMUVB_003/summary Date last accessed: February 2020.

7 Jaakkola K, Saukkoriipi A, Jokelainen J, et al. Decline in temperature and humidity increases the occurrence of influenza in cold climate. Environ Health 2014; 13: 22.

8 Chan KH, Peiris JS, Lam SY, et al. The effects of temperature and relative humidity on the viability of the SARS coronavirus. Adv Virol 2011; 2011: 734690.

9 Aranow C. Vitamin D and the immune system. J Investig Med 2011; 59: 881-886.

10 Duan SM, Zhao XS, Wen RF, et al. Stability of SARS coronavirus in human specimens and environment and its sensitivity to heating and UV irradiation. Biomed Environ Sci 2003; 16: 246-255.

11 Alshukairi AN, Zheng J, Zhao J, et al. High prevalence of MERS-CoV infection in camel workers in Saudi Arabia. mBio 2018; 9: e01985-18. 